\title{
Cyclotrimerization of 3-R-1,2,4-Triazin-5(4H)-ones with Cyclic Ketones
}

\author{
Ilya N. Egorova ${ }^{\text {a }}$ Igor S. Kovalev ${ }^{\mathrm{a}}$, Vladimir L. Rusinov ${ }^{\mathrm{a}}$, and Oleg N. Chupakhin ${ }^{\mathrm{a}, \mathrm{b}}$ \\ a Department of Organic Chemistry, Ural Federal University, Mira St. 19, 620002 Ekaterinburg, \\ Russia \\ ${ }^{b}$ Institute of Organic Synthesis of Russian Academy of Sciences, S. Kovalevskoy St., 22, \\ 620041 Ekaterinburg, Russia
}

Reprint requests to Dr. Ilya Egorov. Fax: +7(343)3740458. E-mail: i.n.egorov@gmail.com

Z. Naturforsch. 2010, 65b, 1359-1362; received July 21, 2010

\begin{abstract}
New heterocyclic tetracyclic systems were synthesized. Interaction between 3- $R$-1,2,4-triazin$5(4 H)$-ones and cyclic ketones under acidic conditions leads to the formation of zwitterion derivatives of 5,6,7,8,9,10,11,12-octahydro-[1,2,4]triazino[1,6- $f$ ]phenanthridine and 1,2,3,6,7,8-hexahydro-bicyclopenta $[b, d]$ pyrido[ $[1,2-f][1,2,4]$ triazine.
\end{abstract}

Key words: Cycloaddition, Cyclic Ketones, Pyridines, 1,2,4-Triazines

\section{Introduction}

Annelated pyridines are widespread in naturally occurring organic compounds [1], and they have broad application in pharmaceuticals [2]. The first tricyclic pyridines, 1,2,3,6,7,8-hexahydrobicyclopenta $[b, d]$ pyridine and 1,2,3,4,7,8,9,10-octahydrophenanthridine were synthesized by Chichibabin by condensation of an ammonia and formaldehyde mixture with cyclopentanone and cyclohexanone, respectively [3]. In this work the possibility of introducing a heterocycle with a double $\mathrm{C}=\mathrm{N}$ bond in the reaction instead of an ammonia and formaldehyde mixture is investigated.

\section{Results and Discussion}

It is known that 3-R-1,2,4-triazin-5(4H)-ones show high reactivity toward $\mathrm{C}$-nucleophiles whereby addition products are typically obtained [4]. The reaction of 3-R-1,2,4-triazin-5(4H)-ones $\mathbf{1 a}-\mathbf{c}$ with cyclic ketones $\mathbf{2 a}, \mathbf{2 b}$ in acidic media leads to the tetracyclic compounds 4-7 [5] (Scheme 1). These compounds are the products of condensation of one molecule of $\mathbf{1 a}-\mathbf{c}$ with two molecules of the cyclic ketones $\mathbf{2 a}, \mathbf{2 b}$. Using the more active nucleophile 1-cyclopent-1-enyl-pyrrolidine (3) instead of the cyclic ketone $2 \mathbf{a}$ under the same conditions leads to the same condensation product 4 (yield: 15\%) (Scheme 1). The molecular structure of compound $\mathbf{5}$ is shown in Fig. 1.

The distribution of charges in zwitterions 4-7 was assigned on the basis of literature data for pyrido[2,1-

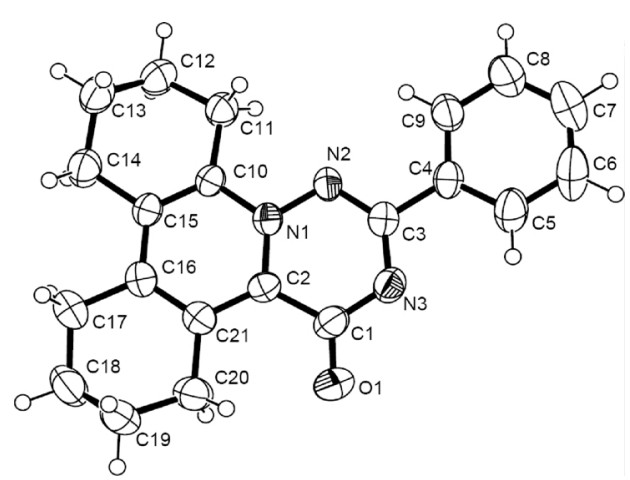

Fig. 1. ORTEP diagram of the molecular structure of $\mathbf{5}$ in the crystal.

f]-1,2,4-triazinium-4-olates which could be regarded to have the base structure for the synthesized polycyclic compounds [5].

The proposed reaction mechanism involves the formation of nucleophilic addition product $\mathbf{A}$ in the first stage, with subsequent addition of one more molecule of cyclic ketone. Compound $\mathbf{B}$ is the result of an elimination of a water molecule and oxidation, leading to products 4-7 (Scheme 2).

\section{Conclusion}

In this work we demonstrated that 3-R-1,2,4-triazin$5(4 H)$-ones can be successfully used as the source of a $\mathrm{C}=\mathrm{N}$ component in the condensation reaction with cyclic ketones to yield tetracyclic pyridine systems. 


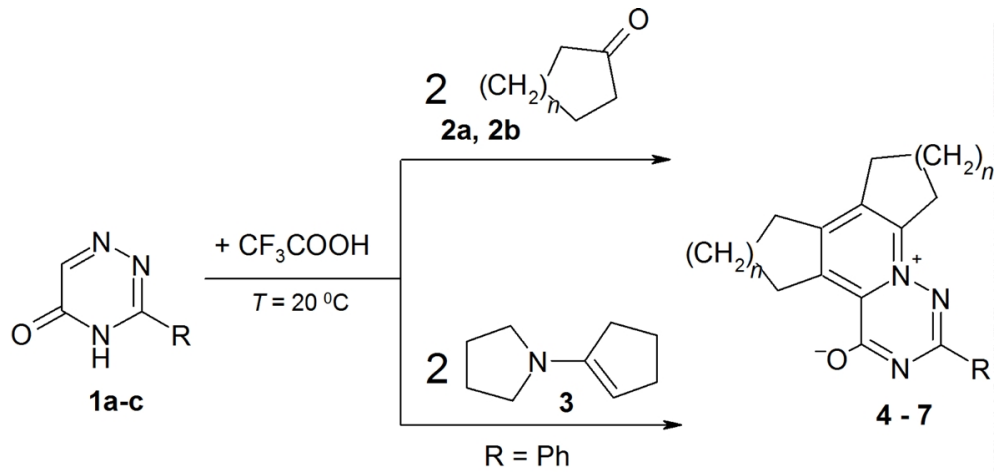

\begin{tabular}{lllll} 
1a: $\mathrm{R}=\mathrm{Ph}$ & $\mathbf{2 a}: n=1$ \\
1b: $\mathrm{R}=4-\mathrm{CIPh}$ & $\mathbf{2 b}: n=2$ & & & \\
1c: $\mathrm{R}=\mathrm{SCH}_{3}$ & & & & \\
\hline Product & $\mathrm{R}$ & $n$ & Solvent & Yield (\%) \\
\hline $\mathbf{4}$ & $\mathrm{Ph}$ & 1 & $\mathrm{DMF}$ & 30 \\
$\mathbf{4}$ & $\mathrm{Ph}$ & 1 & $\mathrm{CH}_{2} \mathrm{Cl}_{2}$ & 16 \\
$\mathbf{5}$ & $\mathrm{Ph}$ & 2 & $\mathrm{DMF}$ & 44 \\
$\mathbf{6}$ & $4-\mathrm{ClPh}$ & 1 & $\mathrm{DMF}$ & 23 \\
$\mathbf{7}$ & $\mathrm{SCH}_{3}$ & 1 & $\mathrm{DMF}$ & 25 \\
\hline
\end{tabular}

Scheme 1.

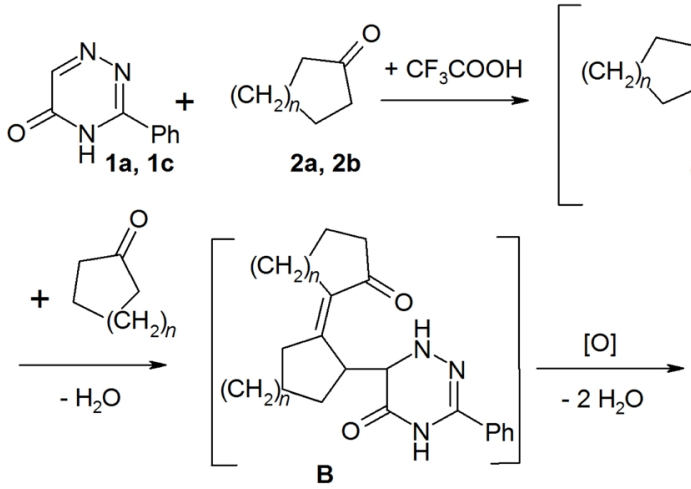

\section{Experimental Section}

3- $R$-1,2,4-triazin-5(4H)-ones 1a, 1b [6], 1c [7] were synthesized by known methods, other starting materials are commercially available. ${ }^{1} \mathrm{H}$ and ${ }^{13} \mathrm{C}$ NMR spectra were recorded on a Bruker DRX-400 spectrometer using TMS as internal standard. The mass analyzer was a Bruker Daltonics (Bremen, Germany) MicrOTOF-Q II mass spectrometer with an electrospray ionization source, a 6-port divert valve and a syringe pump kd Scientific with a flow rate of $180 \mu \mathrm{L} \mathrm{h}^{-1}$.

\section{General procedure for the synthesis of $4-7$}

Trifluoroacetic acid $(100 \mu \mathrm{L})$ was added to a suspension of 3- $R$-1,2,4-triazin-5(4H)-one (200 mg, $1.15 \mathrm{mmol})$ in $3 \mathrm{~mL}$ of solvent with stirring. To the resulting solution was added the corresponding cyclic ketone $\mathbf{2 a}, \mathbf{2 b}$ (or 1-cyclopentenyl- pyrrolidine (3)) (2.3 mmol). The reaction mixture was stirred for $7 \mathrm{~d}$ in an open flask under ambient conditions. The formed sediment was filtered off and recrystallized from DMF. The residual solution after filtration was evaporated, and an additional quantity of product was isolated with the help of column chromatography with $\mathrm{CHCl}_{3}$-EtOH $(99: 1)$ as eluent.

\section{2-Phenyl-1,2,3,6,7,8-hexahydrodicyclopenteno[b,d]pyrido} [1,2-f][1,2,4]triazin-11-ium-4-olate (4)

M.p. $>300{ }^{\circ} \mathrm{C} .-{ }^{1} \mathrm{H}$ NMR $\left(400 \mathrm{MHz}, \mathrm{CDCl}_{3}\right): \delta=$ 2.29-2.20 (m, 2H, $\left.\mathrm{CH}_{2}\right), 2.41-2.31\left(\mathrm{~m}, 2 \mathrm{H}, \mathrm{CH}_{2}\right), 3.02(\mathrm{t}$, $\left.J=7.7 \mathrm{~Hz}, 2 \mathrm{H}, \mathrm{CH}_{2}\right), 3.13\left(\mathrm{t}, J=7.6 \mathrm{~Hz}, 2 \mathrm{H}, \mathrm{CH}_{2}\right), 3.49$ (t, $\left.J=7.6 \mathrm{~Hz}, 2 \mathrm{H}, \mathrm{CH}_{2}\right), 3.59\left(\mathrm{t}, J=7.7 \mathrm{~Hz}, 2 \mathrm{H}, \mathrm{CH}_{2}\right)$, $7.54-7.39$ (m, 3H, Ph), 8.26-8.28 (m, 2H, Ph). $-{ }^{13} \mathrm{C} \mathrm{NMR}$ $\left(100 \mathrm{MHz}, \mathrm{CDCl}_{3}\right): \delta=22.0,24.5,30.4,31.2,31.4,34.0$, 
127.9, 128.0, 130.0, 130.9, 135.5, 139.2, 142.9, 150.6, 153.0, 162.6, 165.6. - ESI-MS: $m / z=310.1536$ (calcd. 310.1527 for $\mathrm{C}_{19} \mathrm{H}_{17} \mathrm{~N}_{3} \mathrm{OLi},[\mathrm{M}+\mathrm{Li}]^{+}$).

\section{2-Phenyl-5,6,7,8,9,10,11,12-octahydro[1,2,4]triazino [1,6-f]phenantridin-13-ium-4-olate (5)}

M. p. $>300{ }^{\circ} \mathrm{C} .-{ }^{1} \mathrm{H} \mathrm{NMR}\left(400 \mathrm{MHz}, \mathrm{CDCl}_{3}\right): \delta=1.74-$ $1.85\left(\mathrm{~m}, 4 \mathrm{H}, 2 \mathrm{CH}_{2}\right), 1.88-1.94\left(\mathrm{~m}, 2 \mathrm{H}, \mathrm{CH}_{2}\right), 1.95-2.02$ $\left(\mathrm{m}, 2 \mathrm{H}, \mathrm{CH}_{2}\right), 2.67\left(\mathrm{t}, J=6.1 \mathrm{~Hz}, 2 \mathrm{H}, \mathrm{CH}_{2}\right), 2.71(\mathrm{t}, J=$ $\left.6.2 \mathrm{~Hz}, 2 \mathrm{H}, \mathrm{CH}_{2}\right), 3.35\left(\mathrm{t}, J=6.2 \mathrm{~Hz}, 2 \mathrm{H}, \mathrm{CH}_{2}\right), 3.61(\mathrm{t}$, $\left.J=6.2 \mathrm{~Hz}, 2 \mathrm{H}, \mathrm{CH}_{2}\right), 7.38-7.46(\mathrm{~m}, 3 \mathrm{H}, \mathrm{Ph}), 8.36-8.38$ $(\mathrm{m}, 2 \mathrm{H}, \mathrm{Ph}) .-{ }^{13} \mathrm{C}$ NMR $\left(100 \mathrm{MHz}, \mathrm{CDCl}_{3}\right): \delta=21.0,21.3$, 21.5, 21.9, 26.3, 27.2, 27.6, 28.8, 128.0, 128.0, 130.8, 132.2, 135.8, 136.3, 143.4, 146.9, 161.5, 166.5. - ESI-MS: $m / z=$ 332.1758 (calcd. 332.1757 for $\mathrm{C}_{21} \mathrm{H}_{22} \mathrm{~N}_{3} \mathrm{O},[\mathrm{M}+\mathrm{H}]^{+}$).

\section{2-(4-Chlorophenyl)-1,2,3,6,7,8-hexahydrodicyclopenteno-} [b,d]pyrido[1,2-f][1,2,4]triazin-11-ium-4-olate (6)

M.p. $>300{ }^{\circ} \mathrm{C} .-{ }^{1} \mathrm{H} \mathrm{NMR}\left(400 \mathrm{MHz}, \mathrm{CDCl}_{3}\right): \delta=2.21-$ $2.28\left(\mathrm{~m}, 2 \mathrm{H}, \mathrm{CH}_{2}\right), 2.41-2.34\left(\mathrm{~m}, 2 \mathrm{H}, \mathrm{CH}_{2}\right), 2.94(\mathrm{t}, J=$ $\left.7.7 \mathrm{~Hz}, 2 \mathrm{H}, \mathrm{CH}_{2}\right), 2.95\left(\mathrm{t}, J=3.8 \mathrm{~Hz}, 2 \mathrm{H}, \mathrm{CH}_{2}\right), 3.07(\mathrm{t}, J=$ $\left.7.6 \mathrm{~Hz}, 2 \mathrm{H}, \mathrm{CH}_{2}\right), 3.47$ (t, $\left.J=7.7 \mathrm{~Hz}, 2 \mathrm{H}, \mathrm{CH}_{2}\right), 3.70$ (t, $J=$ $\left.7.6 \mathrm{~Hz}, 2 \mathrm{H}, \mathrm{CH}_{2}\right), 7.34(\mathrm{~d}, J=8.6 \mathrm{~Hz}, 2 \mathrm{H}, 4-\mathrm{ClPh}), 8.26$ (d, $J=8.6 \mathrm{~Hz}, 2 \mathrm{H}, 4-\mathrm{ClPh}) .-{ }^{13} \mathrm{C} \mathrm{NMR}\left(100 \mathrm{MHz}, \mathrm{CDCl}_{3}\right)$ : $\delta=22.1,24.7,30.5,31.3,31.6,34.1,128.2,129.4,130.3$, 134.1, 137.1, 139.2, 143.2, 150.7, 153.2, 162.0, 165.7. - ESIMS: $m / z=338.1059$ (calcd. 338.1055 for $\mathrm{C}_{19} \mathrm{H}_{17} \mathrm{ClN}_{3} \mathrm{O}$, $\left.[\mathrm{M}+\mathrm{H}]^{+}\right)$.

2-Methylthio-1,2,3,6,7,8-hexahydrodicyclopenteno[b,d]pyrido[1,2-f][1,2,4]triazin-11-ium-4-olate (7)

M. p. $=261-262{ }^{\circ} \mathrm{C} .-{ }^{1} \mathrm{H} \mathrm{NMR}\left(400 \mathrm{MHz}, \mathrm{CDCl}_{3}\right): \delta=$ 2.19-2.27 (m, 2H, $\left.\mathrm{CH}_{2}\right), 2.30-2.38\left(\mathrm{~m}, 2 \mathrm{H}, \mathrm{CH}_{2}\right), 2.50$ $\left(\mathrm{s}, 3 \mathrm{H}, \mathrm{SCH}_{3}\right), 2.96\left(\mathrm{t}, J=7.8 \mathrm{~Hz}, 2 \mathrm{H}, \mathrm{CH}_{2}\right), 3.08(\mathrm{t}, J=$ $\left.7.6 \mathrm{~Hz}, 2 \mathrm{H}, \mathrm{CH}_{2}\right), 3.33\left(\mathrm{t}, J=7.8 \mathrm{~Hz}, 2 \mathrm{H}, \mathrm{CH}_{2}\right), 3.62(\mathrm{t}$, $\left.J=7.6 \mathrm{~Hz}, 2 \mathrm{H}, \mathrm{CH}_{2}\right) .-{ }^{13} \mathrm{C} \mathrm{NMR}\left(100 \mathrm{MHz}, \mathrm{CDCl}_{3}\right): \delta=$
13.5, 22.1, 24.7, 30.5, 31.2, 31.5, 34.0, 129.4, 139.2, 143.3, 149.4, 152.5, 162.8, 172.3. - ESI-MS: $\mathrm{m} / z=274.1009(\mathrm{calcd}$. 274.1009 for $\left[\mathrm{C}_{14} \mathrm{H}_{16} \mathrm{~N}_{3} \mathrm{OS}\right]^{+},[\mathrm{M}+\mathrm{H}]^{+}$).

\section{X-Ray structure determination of $\mathbf{5}$}

$\mathrm{X}$-Ray structure analysis including data collection, cell refinement and data reduction was carried out with an Oxford Diffraction Xcalibur S CCD diffractometer using the CRYSALISPRO software package [8]. X-Ray data collection was carried out at 295(2) K with graphite-monochromatized $\operatorname{Mo} K_{\alpha}$ radiation $(\lambda=0.71073 \AA)$. The structure was solved with Direct Methods using SHELXS-97 [9] and refined by full-matrix least-squares procedures on $F^{2}$ with SHELXL97 [9]. Non-H atoms were refined anisotropically, hydrogen atoms were placed in idealized positions and were constrained to ride on their parent atoms.

Crystal data for 5: $\mathrm{C}_{21} \mathrm{H}_{21} \mathrm{~N}_{3} \mathrm{O}, M_{\mathrm{r}}=331.41$, crystal size $=0.49 \times 0.48 \times 0.04 \mathrm{~mm}^{3}$, monoclinic crystal system, space group $P 2{ }_{1} / n, a=8.2604(12), b=20.9831(18), c=$ 10.0864(17) $\AA, \beta=111.360(13)^{\circ}, V=1628.2(4) \AA^{3}, Z=4$, $\rho_{\text {calc }}=1.35 \mathrm{~g} \mathrm{~cm}^{-3}, F(000)=704, \mu=0.1 \mathrm{~mm}^{-1}$, refls. measured $=6038$, refls. unique $=3216, R_{\text {int }}=0.0548$, refls. observed $[I \geq 2 \sigma(I)]=1385$, parameters refined $=226, R 1=$ $0.0453, w R 2=0.0638$ for $I \geq 2 \sigma(I), R 1=0.1272, w R 2=$ 0.0699 for all data, $G o F=1.003$; residual electron density (min/max): $-0.14 / 0.13$ e $\AA^{-3}$. Comment: The low ratio of observed to unique reflections was caused by the weakly diffracting crystal due to the small crystal size.

CCDC 777392 contains the supplementary crystallographic data for this paper. These data can be obtained free of charge from the Cambridge Crystallographic Data Centre via www.ccdc.cam.ac.uk/data_request/cif.

\section{Acknowledgement}

We are pleased to thank Dr. P. Slepukhin for assistance with the X-ray analysis.
[1] a) T. D. Bailey, G. L. Goe, E. F. V. Scriven, Chem. Heterocycl. Compd. 1984, 14 (Pyridine and Its Derivatives, Part 5), 1-252; b) R. P. Thummel, Chem. Heterocycl. Compd. 1984, 14 (Pyridine and Its Derivatives, Part 5), $253-445$; c) R. Kumar, R. Chandra, Adv. Heterocycl. Chem. 2001, 78, 269-313;

[2] a) D. S. Pisoni, J. S. Costa, D. Gamba, C. L. Petzhold, A. C. A. Borges, M. A. Ceschi, P. Lunardi, C. A. S. Gonçalves, Eur. J. Med. Chem. 2010, 45, 526-535; b) L. Pasumansky, A. R. Hernańdez, S. Gamsey, C. T. Goralski, B. Singaram, Tetrahedron Lett. 2004, 45, 6417-6420; c) J. H. Hutchinson, W. Halczenko, K. M. Brashear, M. J. Breslin, P. J. Coleman, L. T. Duong,
C. Fernańdez-Metzler, M. A. Gentile, J.E. Fisher, G. D. Hartman, J. R. Huff, D. B. Kimmel, C. T. Leu, R. S. Meissner, K. Merkle, R. Nagy, B. Pennypacker, J. J. Perkins, T. Prueksaritanont, G. A. Rodan, S. L. Varga, G. A. Wesolowski, A. E. Zartman, S. B. Rodan, M. E. Duggan, J. Med. Chem. 2003, 46, 4790-4798; d) R. S. Meissner, J.J. Perkins, L. T. Duong, G. D. Hartman, W.F. Hoffman, J. R. Huff, N. C. Ihle, C. T. Leu, R. Nagi, A. Naylor-Olsen, G. A. Rodan, S. B. Rodan, D. B. Whitman, G. A. Wesolowski, M. E. Duggan, Bioorg. Med. Chem. Lett. 2002, 12, 25-29; e) A. Cappelli, M. Anzini, S. Vomero, L. Canullo, L. Mennuni, F. Makovec, E. Doucet, M. Hamon, M. C. Menziani, 
P. G. De Benedetti, G. Bruni, M. R. Romeo, G. Giorgi, A. Donati, J. Med. Chem. 1999, 42, 1556-1575.

[3] A.E. Chichibabin, Bull. Soc. Chim. Fr. 1939, 6, $522-$ 533.

[4] a) V. L. Rusinov, G. V. Zyryanov, T. L. Pilitcheva, O. N. Chupakhin, H. Neunhoeffer, J. Heterocyclic Chem. 1997，34，1013-1019; b) O.N. Chupakhin, G.L. Rusinov, D. G. Beresnev, H. Neunhoeffer, J. Heterocyclic Chem. 1997, 34, 573-578; c) D. G. Beresnev, N. A. Itsikson, O. N. Chupakhin, V. N. Charushin, M. I. Kodess, A. I. Butakov, G. L. Rusinov, Y. Y. Morzherin, A. I. Konovalov, I. S. Antipin, J. Org. Chem. 2006, 71, $8272-8275$.

[5] a) P. Molina, A. Tarraga, M. J. Vilaplana, E. Hurtado, M. Lorenzo, J. Chem. Soc., Perkin Trans. I 1983,
1395 - 1399; b) S. Batori, Zs. Juhasz-Riedl, P. Sandor, A. Messmer, J. Heterocyclic Chem. 1986, 23, $375-$ 380; c) S. Batori, A. Messmer, J. Heterocyclic Chem. 1988, 25, $437-444$.

[6] V. Uchutilova, P. Fiedler, M. Prystas, J. Gut, Collect. Czech. Chem. Commun. 1971, 36, 1955-1963.

[7] L. Heinisch, J. Prakt. Chem. 1974, 316, 667-678.

[8] CRYsAlysPro, CRysAlysRed (version 1.171.31. 8). Oxford Diffraction Ltd., Abingdon, Oxfordshire (England) 2007.

[9] G. M. Sheldrick, SHELXS/L-97, Programs for Crystal Structure Determination, University of Göttingen, Göttingen (Germany) 1997. See also: G. M. Sheldrick, Acta Crystallogr. 1990, A46, 467-473; ibid. 2008, A64, 112 - 122. 